\title{
Healthcare workers' clinical knowledge on maternal and newborn care in Ethiopia: findings from 2016 national EmONC assessment
}

Theodros Getachew Zemedu ${ }^{1,2^{*}}$ (D) Aster Teshome ${ }^{3}$, Yared Tadesse $^{3}$, Abebe Bekele $^{1}$, Emily Keyes $^{4}$, Patricia Bailey ${ }^{4}$ and Ana Lorena Ruano ${ }^{4}$

\begin{abstract}
Background: Improving maternal and newborn health indicators are key if Ethiopia is to achieve the Sustainable Development Goals. To do so, women need access to skilled attendance at birth and emergency obstetric and newborn care. To maximize their impact, understanding gaps in workers' knowledge is required to remedy the weakness. This assessment determines knowledge levels of clinical management of maternal and newborn healthcare and factors that influence knowledge.
\end{abstract}

Methods: This study used data from the National Emergency Obstetric and Neonatal Care assessment conducted in 2016. Provider knowledge for MNH was assessed by interviewing providers. Respondents were scored on each question by calculating the number of correct responses provided out of the total possible answers, and standardizing this to a scale of 100 . Mixed linear regression was used to determine individual and contextual factors associated with the score.

Results: A total of 3800 interviews with complete data were included in this study. Most respondents were diploma midwives (73\%), BSc midwives (11\%) and diploma nurses (10\%). On average, midwives scored 60 out of 100 on the question regarding the primary aspects of focused antenatal care and elements of a birth plan. Half of the midwives and health officers, and one-third of nurses knew to provide a loading dose of magnesium sulphate. Midwives scored $90 \%$ on the steps of active management of third stage of labor. In the mixed linear regression, working in a private for profit facility, health center/clinic, rural area, or in a facility with a protocol on referral/ counter referral predicted lower knowledge scores. More positive scores were associated with work environments that had a computer, internet, and protocols on safe abortion care, management of selected obstetric topics, integrated management of pregnancy, childbirth, postnatal, and newborn, care for low birth weight including kangaroo mother care, and treatment of infection in young infants.

Conclusion: With regard to most knowledge related questions, health officers and midwives scored similarly. Providers scored substantially better on routine intrapartum and newborn care than on aspects related to care for complications. A substantial proportion of providers indicated that they would never give a loading dose of magnesium sulphate.

Keywords: Knowledge, Maternal, Newborn, Ethiopia

\footnotetext{
* Correspondence: tedi.getachew@yahoo.com

${ }^{1}$ Health System and Reproductive Health Research Directorate, Ethiopian

Public Health Institute, Addis Ababa, Ethiopia

${ }^{2}$ College of Medicine and Health Sciences, Institute of Public Health,

University of Gondar, Gondar, Ethiopia

Full list of author information is available at the end of the article
}

(c) The Author(s). 2019 Open Access This article is distributed under the terms of the Creative Commons Attribution 4.0 International License (http://creativecommons.org/licenses/by/4.0/), which permits unrestricted use, distribution, and reproduction in any medium, provided you give appropriate credit to the original author(s) and the source, provide a link to the Creative Commons license, and indicate if changes were made. The Creative Commons Public Domain Dedication waiver (http://creativecommons.org/publicdomain/zero/1.0/) applies to the data made available in this article, unless otherwise stated. 


\section{Background}

Although there has been substantial progress in reducing maternal and neonatal mortality in the last 20 years, it is estimated that about 287,000 women and 2.6 million newborns die of pregnancy and birth-related complications each year [1]. About $85 \%$ of the global burden of maternal and neonatal deaths happen in low and middle-income countries (LMICs), with a disproportionate share of them taking place in sub-Saharan Africa [2]. Ethiopia is no exception, and country's estimated maternal mortality ratio (MMR) is 676 deaths per 100,000 live births, and the neonatal mortality rate (NMR) is 37 deaths per 1000 live births [3]. Among the main causes of maternal death are postpartum hemorrhage, hypertensive disorders of pregnancy, infections, and obstructed labor. For newborns, they are prematurity, intrapartum complications and infections [4]. Many of these deaths could be averted through access to skilled institutional care.

Improving maternal and newborn health indicators are key if Ethiopia is to meet the Sustainable Development Goals (SDGs). To achieve this, the country needs to ensure access to skilled attendance at birth and emergency obstetric and newborn care (EmONC) for all women and infants who need it. EmONC interventions are a cost-effective way to reduce maternal and neonatal morbidity and mortality in poor resource settings [5], and basic EmONC (BEmONC) alone can avert 40\% of intrapartum related neonatal deaths, as well as a significant proportion of maternal mortality [6]. According to the health sector transformation plan, (HSTP) Ethiopia has set a target of reducing the country's MMR to 199/100, 000 and the NMR to $12 / 1000$ by 2020 [4]. To attain these goals, the country has intensified EmONC capacity building trainings, which have been implemented since 1998. The goal of these training sessions, which targets nurses, midwives and health officers, is to address the knowledge and skill gaps that providers face during complicated deliveries.

Despite the favorable impact on maternal and newborn survival that EmONC interventions have, current understanding of healthcare worker knowledge remains limited throughout the region. In Mali and Kenya, primary healthcare workers reported little knowledge or competency of EmONC, particularly when it comes to hypertensive patients, postpartum infections and obstetric emergencies in general [7-9]. Insufficient knowledge regarding the diagnoses of emergency conditions, like postpartum hemorrhage (PPH) and birth asphyxia, was higher among nurses and midwives from Addis Ababa [5]. However, these studies focused on specific cadres of healthcare workers and were carried out in urban settings or based on small samples. Currently, we have limited information on knowledge among all healthcare cadres in all regions of Ethiopia. Therefore, the purpose of this paper is to evaluate gaps related to clinical knowledge on routine maternal and newborn care, treatment of complications and the factors that influence knowledge.

\section{Methods \\ Study setting}

We used cross-sectional data from the National Emergency Obstetric and Neonatal Care assessment, a collaboration between the Averting Maternal Mortality and Disability program (AMDD) and the Ethiopian Institute of Public Health (EPHI). Data were collected between the months of May and October of 2016 in all 9 regions and in the 2 city administrations of the country.

Questionnaires were paper-based, followed by performing data entry later in the day in the field. This process was called CAFE (computer-assisted field editing) and each team had one tablet. Face-to-face interviewing was used to collect the data. The data were sent to the Central Office using the Internet File Streaming System (IFSS). These files were extracted, reviewed, and checked for errors and inconsistencies. Secondary editing was done by two data managers located at EPHI and if errors or inconsistencies were found, teams were asked to correct and resend the data.

\section{Participants}

In total, the survey included 3804 health facilities. All eligible public hospitals (referral, general, primary) and health centers, and all eligible for-profit and not-forprofit private facilities (hospitals, Maternal and Child Health $(\mathrm{MCH})$, specialty centers, $\mathrm{MCH}$ specialty clinics, and higher clinics) were included in the study. Eligibility was determined by three characteristics: 1) health facilities classified as higher clinic or above as per the regulation set by the Food, Medicine and Health Care Administration and Control Authority of Ethiopia (FMHACA); and 2) the facility reported having attended births in the last 12 months; and 3) the facility was deemed functional, i.e. it was not under construction and was at least minimally operational. The target population for this study consisted of all health worker cadres. The target population for this study consisted of a single functioning birth attendant per facility. Three selection criteria based on practicality were applied: the individual who had attended the largest number of deliveries in the last month, regardless of health worker cadre, and who was physically present and consented during the data collection team visit to the facility. If no births had been reported in the facility in the previous 30 days, the last 60 days were considered. Thus, the pool of birth attendants consisted largely of mid-level professionals since doctors and specialists are generally not 
found in health centers and health centers were far more numerous than hospitals. The data collection teams were health workers trained for two week on the data collection tools. Data collection occurred from May to November 2016.

\section{Measurement}

Based on the set of knowledge questions administered to healthcare workers, extracted from the national clinical guidelines for Emergency Obstetric Care that covered a range of maternal and newborn topics, such as routine and emergency care during pregnancy, intrapartum care, essential newborn care, and care for sick newborns, a knowledge level score was generated. Average summary scores were then calculated for each specific question included in the topic area. Each knowledge question had multiple "correct" answers; that is, answers that respondents were expected to provide spontaneously. If a correct answer was not offered spontaneously, the interviewer coded the response as "not mentioned." If a spontaneous answer did not appear as one of the precoded options, it was not taken into consideration for scoring purposes. Respondents were scored on each question by calculating the number of correct responses mentioned out of the total possible, and standardizing this to a scale of 100 . Antenatal care, for example, was represented by three questions, the first of which asked respondents to list the components of focused antenatal care that had eight possible responses. In order to handle confounding effects, a mixed-effect linear regression model was used to determine individual and contextual factors associated with the knowledge score. The regression coefficient tells us how much the summary knowledge score is expected to increase when the independent variable increases by one, holding all other independent variables constant. In addition to the univariate model, we fitted three models. Model I was fitted on facility level factors, Model II on provider-level factors, and Model III included both facility and provider level factors. Akaike's information criterion (AIC) and Bayesian information criterion (BIC) were run to measure the models fit and complexity. For the given models fitted on the same data, the model with the smallest value of the information criterion is considered to be the best.

\section{Results}

\section{Socio demographic characteristics of health care providers}

From the possible maximum total of 3804 healthcare providers, 3800 interviews with complete data were included in this study, for a response rate of $99.9 \%$. Most respondents were diploma midwives (73\%), followed by BSc midwives (11\%), and diploma nurses (10\%). Most respondents were female (63\%), though the sex distribution varied by cadre, facility type and managing authority. The average respondent was 26 years old, had been posted to the current facility for 2 years, and had been practicing with current qualification for just over 3 years. Midwives reported attending around 21 deliveries per month, exceeded by 4 medical doctors who reported delivering 24 deliveries per month. The monthly average of deliveries conducted by nurses was between 11 and 12, followed by health officers who attended on average 9 deliveries per month (Table 1).

\section{Knowledge of care during pregnancy, intrapartum, abortion and violence, and newborn care}

Knowledge score were, aggregated by cadre, and presented in Table 2. Due to small number of respondents for medical doctors and other health professional category, results were omitted from this table.

Health workers scored above 50 on two out of the three antenatal questions.

Health workers tended to score higher on questions related to routine intrapartum or newborn care than they did on care for maternal or newborn complications. Scores were highest for questions regarding the timing of a newborn's first bath (score of 95) and the steps of Active Management of Third Stage of Labour (AMTSL) (score of 80) and lowest for which women require a special care plan (score of 35) or care for low birth weight newborns (score of 36). Nurses consistently scored lower than health officers and midwives (Table 2).

The overall knowledge score for maternal and newborn health care among midwives, nurses, and health officers were 52, 43, and 54 respectively. In general, midwives and health officers consistently scored higher than nurses in all topic areas of maternal and newborn health care (Fig. 1).

\section{Determinants of health providers' knowledge on maternal and newborn care}

The determinants of health providers' knowledge on maternal and newborn care are presented in Table 3. Model I has the smallest AIC and BIC (Table 3), therefore Model I based on facility level factors had the best fit and was the least complex.

Negative coefficients, indicating a lowering of the knowledge score, were observed when providers worked in a private for-profit facility, in a health center/clinic, in a rural facility, and where there was a protocol on referral and counter referral. On the other hand, positive coefficients were found when providers worked in facilities with a computer, with internet, with protocols on safe abortion care, on management of selected obstetric topics, on integrated management of pregnancy, childbirth, postnatal, and newborn, and on care for Low Birth 
Table 1 Percent distribution of providers according to health worker cadre, facility type, and managing authority, and distribution of providers according to demographic and professional characteristics, ${ }^{1}$ Ethiopia

\begin{tabular}{|c|c|c|c|c|c|c|c|c|}
\hline & \multirow{2}{*}{\multicolumn{2}{|c|}{$\begin{array}{l}\text { Providers } \\
\text { interviewed }\end{array}$}} & \multicolumn{3}{|c|}{ Characteristics } & \multicolumn{3}{|c|}{ Professional experience } \\
\hline & & & \multicolumn{2}{|l|}{ Sex } & \multirow{2}{*}{$\begin{array}{l}\text { Mean } \\
\text { age } \\
\text { (in } \\
\text { years) }\end{array}$} & \multirow{2}{*}{$\begin{array}{l}\text { Mean } \\
\text { number } \\
\text { of } \\
\text { deliveries } \\
\text { attended } \\
\text { in past } \\
\text { month }\end{array}$} & \multirow{2}{*}{$\begin{array}{l}\text { Mean } \\
\text { number } \\
\text { of years } \\
\text { at } \\
\text { current } \\
\text { facility }\end{array}$} & \multirow{2}{*}{$\begin{array}{l}\text { Mean } \\
\text { number of } \\
\text { years since } \\
\text { receiving } \\
\text { professional } \\
\text { qualification }\end{array}$} \\
\hline & $n$ & $\%$ & Female & Male & & & & \\
\hline National & 3800 & 100 & 63 & 37 & 25.7 & 19.3 & 2.0 & 3.2 \\
\hline \multicolumn{9}{|l|}{ Health worker cadre } \\
\hline MD (general practitioner) & 4 & 0 & 50 & 50 & 36.3 & 24.5 & 1.8 & 9.7 \\
\hline Midwife (BSC) & 407 & 11 & 37 & 63 & 25.8 & 21.1 & 1.4 & 2.4 \\
\hline Midwife (Diploma) & 2786 & 73 & 74 & 26 & 25.5 & 20.8 & 1.9 & 3.2 \\
\hline Nurse (BSc) & 86 & 2 & 42 & 58 & 28.1 & 12.1 & 3.2 & 3.7 \\
\hline Nurse (Diploma) & 370 & 10 & 35 & 65 & 26.7 & 11.1 & 2.8 & 4.6 \\
\hline Health officer & 143 & 4 & 25 & 76 & 25.8 & 8.8 & 1.5 & 2.0 \\
\hline Other $^{2}$ & 4 & 0 & 0 & 100 & 32.3 & 1.5 & 1.0 & 3.8 \\
\hline \multicolumn{9}{|l|}{ Facility type } \\
\hline Referral/specialized hospitals & 30 & 1 & 50 & 50 & 27.8 & 25.8 & 3.0 & 4.8 \\
\hline General hospitals & 103 & 3 & 68 & 32 & 28.4 & 16.2 & 3.0 & 5.1 \\
\hline Primary hospitals & 160 & 4 & 48 & 52 & 26.7 & 18.7 & 1.8 & 3.3 \\
\hline MCH specialty centers & 23 & 1 & 87 & 13 & 31.0 & 10.2 & 2.9 & 6.8 \\
\hline Health centers & 3455 & 91 & 64 & 36 & 25.5 & 19.4 & 1.9 & 3.1 \\
\hline MCH specialty clinics & 16 & 0 & 69 & 31 & 28.6 & 7.5 & 1.2 & 4.7 \\
\hline Higher clinics & 13 & 0 & 69 & 31 & 28.3 & 8.6 & 3.8 & 5.5 \\
\hline \multicolumn{9}{|l|}{ Managing authority } \\
\hline Public/government & 3658 & 96 & 63 & 37 & 25.6 & 19.6 & 1.9 & 3.1 \\
\hline Private-for-profit & 83 & 2 & 82 & 18 & 28.5 & 6.4 & 3.2 & 6.7 \\
\hline Private-not-for-profit ${ }^{3}$ & 59 & 2 & 66 & 34 & 29.6 & 12.8 & 3.5 & 5.5 \\
\hline
\end{tabular}

${ }^{1}$ Non-response varies and does not exceed 3.6. Non-responses are excluded

${ }^{2}$ Other health worker cadres include emergency surgical officers and health extension practitioners

${ }^{3}$ Includes NGO, faith-based, or mission facilities

Weight (LBW) infants including Kangaroo Mother Care (KMC) (Table 3).

\section{Discussion}

In this study we aimed to assess the level of health worker knowledge regarding essential standard components of maternal and newborn care. Health officers and midwives scored similarly and higher than nurses on most knowledge questions. Substantial proportion of providers who indicated that they would never give a loading dose of magnesium sulphate. Only average scores were observed regarding health workers' knowledge on routine newborn care. All provider cadres scored substantially better on aspects of routine intrapartum and newborn care than on aspects of care for intrapartum or newborn complications, which might be expected given that the vast majority of the health workers were mid-level practitioners. Nevertheless, all were front-line providers who are faced with maternal and newborn complications, if only to stabilize and refer them to doctors and specialists posted at hospitals. Knowledge levels were higher among providers posted in hospitals, suggesting they might have greater exposure to women and newborns with complications and to more highly skilled staff.

Magnesium sulphate was expected to be provided in all type of cadres under this study, however, there was a substantial proportion of providers who indicated that they would never give a loading dose of magnesium sulphate; among nurses, this was one in five respondents. Magnesium sulphate has been on the World Health Organization's (WHO) essential medicines list since 1996, and it is an affordable drug [10]. However, magnesium sulphate has not achieved widespread usage 
Table 2 Provider knowledge scores (out of 100) related to a set of questions on antenatal care, intrapartum, newborn, postnatal, postpartum, abortion care and violence by health worker cadre, Ethiopia

\begin{tabular}{|c|c|c|c|c|}
\hline & $\begin{array}{l}\text { Midwives } \\
(n=3193)\end{array}$ & $\begin{array}{l}\text { Nurses } \\
(n=456)\end{array}$ & $\begin{array}{l}\text { Health officers } \\
(n=143)\end{array}$ & Total \\
\hline \multicolumn{5}{|l|}{ ANC } \\
\hline Primary aspects of focused antenatal care $(n=8)$ & 58 & 50 & 57 & 55 \\
\hline Elements of a birth plan $(n=5)$ & 59 & 46 & 53 & 53 \\
\hline Women who require a special care plan $(n=10)$ & 32 & 30 & 42 & 35 \\
\hline \multicolumn{5}{|l|}{ Intrapartum } \\
\hline \multicolumn{5}{|l|}{ ROUTINE CARE } \\
\hline Admission and referral requirement before onset of labor $(n=6)$ & 42 & 37 & 48 & 42 \\
\hline Observations to monitor labor progress $(n=10)$ & 75 & 56 & 70 & 67 \\
\hline Steps of active management of the third stage of labor (AMTSL) $(n=3)$ & 90 & 68 & 83 & 80 \\
\hline \multicolumn{5}{|l|}{ CARE FOR COMPLICATIONS } \\
\hline Management principles for women with $\geq 1$ previous caesarean delivery and uterine scar $(n=3)$ & 49 & 42 & 57 & 49 \\
\hline Management principles for women with PROM $(n=5)$ & 38 & 26 & 47 & 37 \\
\hline Management principles for women with heavy bleeding after delivery $(n=10)$ & 52 & 40 & 50 & 47 \\
\hline Loading dose of magnesium sulphate* $(n=4)$ & 52 & 33 & 51 & 45 \\
\hline \multicolumn{5}{|l|}{ Newborn } \\
\hline \multicolumn{5}{|l|}{ ROUTINE CARE } \\
\hline Immediate newborn care $(n=13)$ & 68 & 60 & 66 & 65 \\
\hline Key counselling messages for cord care $(n=4)$ & 51 & 44 & 49 & 48 \\
\hline Timing of first bath $(n=1)$ & 97 & 93 & 94 & 95 \\
\hline \multicolumn{5}{|l|}{ CARE FOR COMPLICATIONS } \\
\hline Care for low birth weight newborns $(<2000 \mathrm{~g})(n=9)$ & 38 & 32 & 39 & 36 \\
\hline Signs and symptoms of newborn infection and sepsis $(n=5)$ & 42 & 36 & 54 & 44 \\
\hline Signs of critical illness for a newborn requiring referral $(n=10)$ & 32 & 31 & 40 & 34 \\
\hline Diagnosis of birth asphyxia $(n=4)$ & 46 & 40 & 51 & 46 \\
\hline Steps of neonatal resuscitation $(n=8)$ & 51 & 39 & 46 & 45 \\
\hline \multicolumn{5}{|l|}{ Postnatal and postpartum } \\
\hline Checks for the baby at a postnatal visit $(n=8)$ & 49 & 44 & 54 & 49 \\
\hline Checks for the mother at a postpartum visit $(n=12)$ & 40 & 32 & 41 & 38 \\
\hline \multicolumn{5}{|l|}{ Abortion and violence } \\
\hline Immediate complications of unsafe abortion $(n=5)$ & 51 & 47 & 60 & 53 \\
\hline Steps to treat woman with complications of an unsafe or incomplete abortion $(n=10)$ & 46 & 40 & 52 & 46 \\
\hline Information to provide to clients treated for an unsafe or incomplete abortion $(n=7)$ & 42 & 37 & 46 & 42 \\
\hline Steps to take when treating a woman who is a victim of rape $(n=8)$ & 35 & 33 & 42 & 37 \\
\hline Total knowledge score (out of 100) & 52 & 43 & 54 & 50 \\
\hline
\end{tabular}

Provider knowledge score for when (under what circumstances) to give a loading dose of magnesium sulphate is not measured among providers who said they would never give the drug ( $12 \%$ of midwives, $20 \%$ of nurses, $13 \%$ of health officers)

Knowledge score on 4 medical doctors and 4 other health professionals were not presented in this table.

in developing countries. This is due to lack of public awareness of the drug, lack of adequate service-provider training, and not all facilities had magnesium sulphate in stock $[11,12]$.

Only average scores were observed regarding health workers' knowledge on routine newborn care. This means that women in the postnatal period may not receive adequate information on immediate newborn care, hygienic cord care, timing of first bath for the newborn, and care for newborns with low birth weight.

A study undertaken in Mali to determine individual and contextual factors associated with emergency obstetric and neonatal care services suggests that the existence of clinical guidelines or protocols is an important factor 


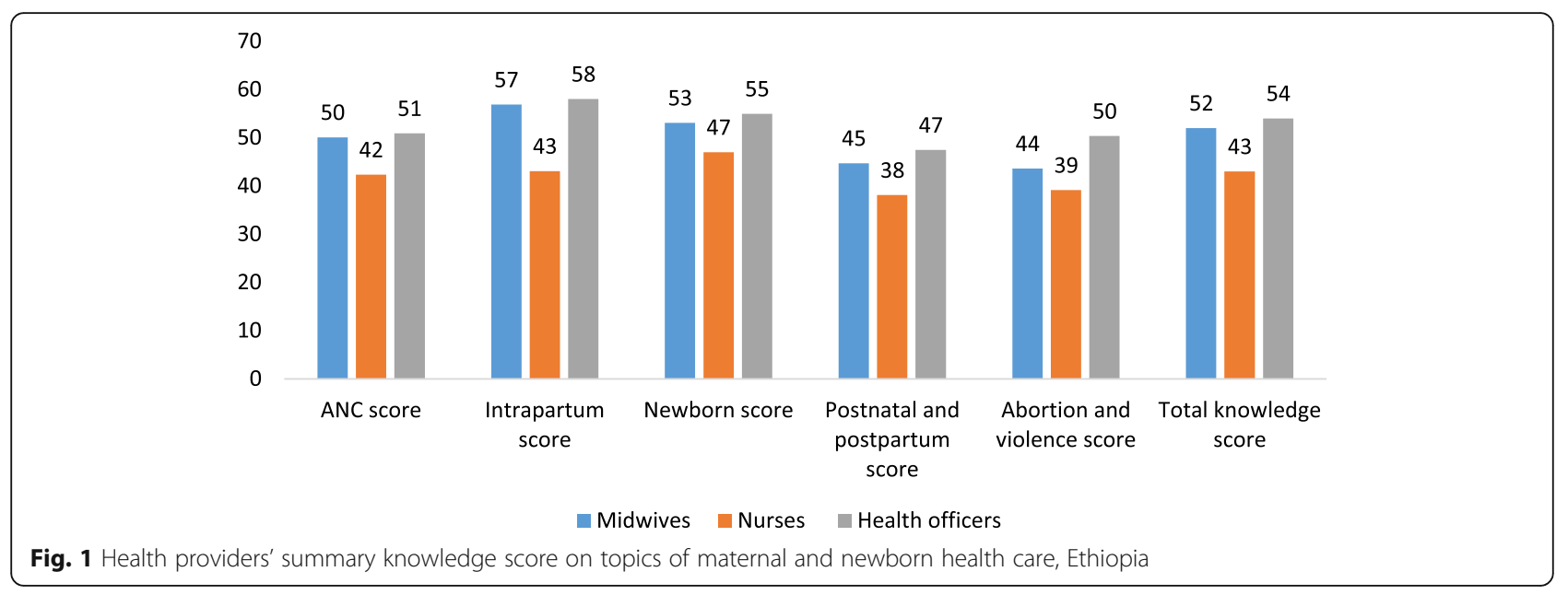

associated with knowledge of healthcare providers [7]. Even if they used small sample size compared with us, similarly, the present study showed a significant positive association between the existence of guidelines in the workplace and knowledge. It could be that healthcare professionals actually read and know the guidelines better when they have them at hand. In contrast with the current study, female gender revealed a significant positive association with provider knowledge in Rwanda where the focuse were the final-year medical students at university suggesting female students had a higher likelihood of demonstrating retention and competency compared with their male counterparts [13]. In a study on knowledge of birth preparedness and complication readiness among doctors, nurses and midwives in Benin, awareness and training on birth preparedness and educational status were significant predictors of knowledge [14]. This study used small sample size compared to our study which is around four hundred health care providers.

The national reach and the large number of health providers included in the study were important strengths of this study. But it was not without some limitations. The assessment was based on respondents' reports, rather than direct observation, which might have led to some reporting error. Most of the questions had multiple correct answers that required spontaneous responses; this may have biased scores towards the lower end because of respondents' fatigue (due to interview or lack of sleep if on night duty), or the interviewer was in a hurry and failed to adequately probe and encourage the provider to think of other responses. If, for example, all questions had been posed as multiple choice answers, the scores might have been higher.

Steps were taken to ensure the quality of the data collected. All data collectors received the same two-week training. Regular supervision of questionnaire completion, along with using electronic data collection approaches contributed to high quality. In addition, the electronic transfer system we utilized regularly reviewed the quality of the data and provided feedback while the data collectors were still in the field.

Our findings suggest that in-service and refresher trainings and dissemination of tailored clinical guidelines for the management of maternal and newborn care are vital to update health workers' knowledge levels and self-confidence in their skills, which may lead to them serving the community better. Since the workers in hospitals had higher scores than workers from health centers, it is recommended that healthcare workers do rotations in hospitals; also, it would be important to ensure access to internet and to clinical guidelines in all facilities.

Although midwives made up the bulk of the sample of respondents, where there are no midwives and nurses or health officers are expected to attend births, we see that nurses were the least knowledgeable. This is both an argument to ensure midwives at every health center and that nurses get more specialized obstetric training. The study compared knowledge scores among midwives, nurses and health officers who have different pre-service training backgrounds. However, during practice all health workers are expected to offer standard care in the provision of maternal and newborn services.

This study have a number of policy implications. Clinical decision making involves combining the knowledge arising from one's clinical expertise that would be improved by provision of guidelines, patient preferences, and research evidence within the context of available resources including computer and internet.

\section{Conclusion}

Health officers and midwives scored similarly on most knowledge questions. All provider cadres scored substantially better on aspects related to routine intrapartum and newborn care than on aspects of complicated 
Table 3 Multivariable mixed-effects linear regression analysis to identify the provider and facility-level determinants of clinical knowledge on maternal and newborn care in Ethiopia $(n=3800)$

\begin{tabular}{|c|c|c|c|c|c|c|c|c|}
\hline \multirow[t]{2}{*}{ Variables } & \multicolumn{2}{|c|}{ Univariate model } & \multicolumn{2}{|l|}{ Model I } & \multicolumn{2}{|l|}{ Model II } & \multicolumn{2}{|c|}{ Model III } \\
\hline & $\beta$ Coef. & $P$-value & $\overline{\beta \text { Coef. }}$ & $P$-value & $\beta$ Coef. & $P$-value & $\beta$ Coef. & $P$-value \\
\hline \multicolumn{9}{|l|}{ Provider-level variables } \\
\hline \multicolumn{9}{|l|}{ Providers cadre } \\
\hline Medical Doctors & 1 & & & & 1 & & 1 & \\
\hline Health Officers & 0.050 & 0.576 & & & 0.090 & 0.299 & 0.082 & 0.340 \\
\hline Midwife BSC and Above & 0.084 & 0.344 & & & 0.131 & 0.125 & 0.109 & 0.203 \\
\hline Midwife diploma & 0.016 & 0.857 & & & 0.073 & 0.389 & 0.061 & 0.470 \\
\hline Nurse BSC & 0.026 & 0.771 & & & 0.097 & 0.262 & 0.079 & 0.364 \\
\hline Nurse diploma & -0.070 & 0.433 & & & 0.002 & 0.978 & -0.007 & 0.937 \\
\hline Total experience & -0.001 & 0.011 & & & 0.000 & 0.132 & 0.000 & 0.167 \\
\hline NO of facilities posted previously & 0.000 & 0.179 & & & 0.000 & 0.769 & 0.000 & 0.785 \\
\hline Experience in current facility & 0.000 & 0.907 & & & 0.000 & 0.958 & 0.000 & 0.946 \\
\hline Providers age & 0.001 & 0.192 & & & 0.000 & 0.543 & 0.000 & 0.874 \\
\hline No. of delivery attended in last month & 0.000 & 0.004 & & & 0.000 & 0.627 & 0.000 & 0.794 \\
\hline \multicolumn{9}{|l|}{ Providers sex } \\
\hline Female & 1 & & & & 1 & & 1 & 1 \\
\hline Male & 0.018 & $<0.001$ & & & 0.028 & $<0.0001$ & 0.029 & $<0.0001$ \\
\hline No. of maternal and newborn services delivered in last 3 month & 0.011 & $<0.001$ & & & 0.003 & $<0.0001$ & 0.006 & $<0.0001$ \\
\hline No. of training received on maternal and newborn services & 0.003 & $<0.001$ & & & 0.254 & $<0.0001$ & 0.003 & $<0.0001$ \\
\hline \multicolumn{9}{|l|}{ Facility-level variables } \\
\hline \multicolumn{9}{|l|}{ Managing authority } \\
\hline Public & 1.000 & & 1.000 & & & & 1.000 & 1.000 \\
\hline Private for profit & 0.002 & 0.892 & -0.049 & $<0.001$ & & & -0.049 & 0.005 \\
\hline Private for non-profit & 0.006 & 0.707 & -0.014 & 0.419 & & & -0.012 & 0.495 \\
\hline \multicolumn{9}{|l|}{ Types of facility } \\
\hline Hospital/MCH specialty center & 1.000 & & 1.000 & & & & 1.000 & 1.000 \\
\hline Health center/clinics & -0.064 & $<0.0001$ & -0.043 & $<0.0001$ & & & -0.023 & 0.054 \\
\hline \multicolumn{9}{|l|}{ Facility location } \\
\hline Urban & 1 & & 1 & & & & 1 & 1 \\
\hline Rural & -0.037 & & -0.019 & $<0.0001$ & & & -0.021 & 0.001 \\
\hline \multicolumn{9}{|l|}{ Availability of computer } \\
\hline No & 1 & & 1 & & & & 1 & 1 \\
\hline Yes & 0.031 & $<0.0001$ & 0.010 & 0.041 & & & -0.001 & 0.917 \\
\hline \multicolumn{9}{|l|}{ Availability of internet } \\
\hline No & 1 & & 1 & & & & 1 & 1 \\
\hline Yes & 0.058 & $<0.0001$ & 0.020 & 0.043 & & & 0.016 & 0.164 \\
\hline \multicolumn{9}{|l|}{ Availability of safe abortion care guideline } \\
\hline No & 1 & & 1 & & & & 1 & 1 \\
\hline Yes & 0.037 & $<0.0001$ & 0.015 & 0.002 & & & -0.005 & 0.400 \\
\hline \multicolumn{9}{|l|}{ Availability of protocol on management of selected obstetric topics } \\
\hline No & 1 & & 1 & & & & 1 & 1 \\
\hline Yes & 0.031 & $<0.0001$ & 0.012 & 0.014 & & & 0.002 & 0.723 \\
\hline
\end{tabular}

Availability of protocol on integrated management of pregnancy, childbirth, postnatal, and newborn 
Table 3 Multivariable mixed-effects linear regression analysis to identify the provider and facility-level determinants of clinical knowledge on maternal and newborn care in Ethiopia $(n=3800)$ (Continued)

\begin{tabular}{|c|c|c|c|c|c|c|c|}
\hline \multirow[t]{2}{*}{ Variables } & \multicolumn{2}{|c|}{ Univariate model } & \multicolumn{2}{|c|}{ Model I } & Model II & \multicolumn{2}{|c|}{ Model III } \\
\hline & $\overline{\beta \text { Coef. }}$ & $P$-value & $\beta$ Coef. & $P$-value & $\beta$ Coef. $\quad P$-value & $\overline{\beta \text { Coef. }}$ & $P$-value \\
\hline No & 1 & & 1 & & & 1 & 1 \\
\hline Yes & 0.029 & $<0.0001$ & 0.011 & 0.030 & & -0.002 & 0.797 \\
\hline \multicolumn{8}{|c|}{ Availability of protocol on care for LBW including KMC } \\
\hline No & 1 & & 1 & & & 1 & 1 \\
\hline Yes & 0.027 & $<0.0001$ & 0.012 & 0.017 & & 0.015 & 0.018 \\
\hline \multicolumn{8}{|c|}{ Availability of protocol on treatment of infection in young infants (IMNCI) } \\
\hline No & 1 & & 1 & & & 1 & 1 \\
\hline Yes & 0.019 & $<0.0001$ & 0.005 & 0.343 & & -0.002 & 0.724 \\
\hline \multicolumn{8}{|c|}{ Availability of protocol on referral and counter referral } \\
\hline No & 1 & & 1 & & & 1 & 1 \\
\hline Yes & 0.011 & 0.028 & -0.012 & 0.018 & & -0.006 & 0.340 \\
\hline \multicolumn{8}{|l|}{ Random effect } \\
\hline Variance (SE) & & & \multicolumn{2}{|c|}{$0.016(0.0004)$} & $0.014(0.0004)$ & \multicolumn{2}{|c|}{$0.014(0.0004)$} \\
\hline \multicolumn{8}{|l|}{ Model Fitness } \\
\hline \multicolumn{2}{|l|}{ Log Likelihood } & & \multicolumn{2}{|c|}{2428.184} & 1469.503 & \multicolumn{2}{|c|}{1483.823} \\
\hline \multicolumn{2}{|l|}{ AIC } & & \multicolumn{2}{|c|}{-4828.368} & -2907.007 & \multicolumn{2}{|c|}{-2911.647} \\
\hline \multicolumn{3}{|l|}{$\mathrm{BIC}$} & \multicolumn{2}{|c|}{-4740.981} & -2816.673 & \multicolumn{2}{|c|}{-2753.589} \\
\hline
\end{tabular}

Model I was fitted on facility level variables, Model II on provider-level variables, and Model III included both facility and provider level variables Bold entries have signficant values at $5 \%$

care. There was a substantial proportion of providers who indicated that they would never give a loading dose of magnesium sulphate; a better understanding of why nurses and others had this response would lead to better care for both the woman and her baby.

In a mixed linear regression, working in a private for profit facility, in health centers/clinics, in rural facilities or in a facility with referral and counter referral guidelines predicted lower knowledge score, while the availability of a computer, the internet, and the existence of protocols, were found to be independent positive predictors of providers' knowledge on maternal and newborn care.

\footnotetext{
Abbreviations

AIC: Akaike Information Criterion; AMDD: Averting Maternal Mortality and Disability program; AMTSL: Active Management of Third Stage of Labour; BIC: Bayesian Information Criterion; EPHI: Ethiopian Institute of Public Health; FMHACA: Food, Medicine and Health Care Administration and Control Authority of Ethiopia; HSTP: Health Sector Transformation Plan; IMNCI: Integrated Management of Newborns and Child Infections; KMC: Kangaroo Mother Care; LBW: Low Birth Weight; LMICs: Low and Middle-Income Countries; MCH: Maternal and Child Health; MMR: Maternal Mortality Rate; NMR: Neonatal Mortality Rate; PPH: Postpartum Hemorrhage SDG: Sustainable Development Goal; WHO: World Health Organization
}

\section{Acknowledgements}

We would like to acknowledge the Ethiopian Public Health Institute (EPHI) for allowing free access of the data and it can also be accessed. We would also like to acknowledge EPHI's health system and reproductive health research directorate team for their criticism of this manuscript during their journal club meetings.

\section{Authors' contributions}

TGZ, AT, YT, PB, EK, ALR, AB conceptualized the design of the study. TGZ participated in data extraction, and analysis; TGZ, AT, YT, PB, EK, ALR, and AB provided review of the methodology and analysis results; All authors contributed to the writing of this paper and all have read and approved the final manuscript.

\section{Funding}

The Bill and Melinda Gates foundation funded the preparation of this research paper through a grant to the Averting Maternal Death and Disability (AMDD) program at Columbia University. This research paper represents the views of the authors and does not represent the views of the funder. The funder had no role in the design, analysis or write-up of this research manuscript.

\section{Availability of data and materials}

The data for this manuscript was provided by the Ethiopian Public Health Institute (EPHI). The primary data is possible to access after getting ethical approval from the EPHI institutional review board. Interested researchers may contact the Director of the Health System and Reproductive Health Research Directorate at the EPHI, Addis Ababa, Ethiopia through email; abebe12

77belay@gmail.com .

\section{Ethics approval and consent to participate}

The primary source of the data was based on the 2016 EmONC assessment that underwent ethical approval through the Ethiopian Public Health Institute's (EPHI) scientific and ethical review board. All selected providers were asked for their written consent to participate. If they refused, no other provider was selected from that facility. No incentive was given for participation in the study. To maintain confidentiality no personal identifier was used and data were maintained on the EPHI password protected server. This further analysis is exempted from review. 


\section{Consent for publication}

Not applicable.

\section{Competing interests}

The authors declared that there is no financial and non-financial competing interest.

\section{Author details}

'Health System and Reproductive Health Research Directorate, Ethiopian Public Health Institute, Addis Ababa, Ethiopia. ${ }^{2}$ College of Medicine and Health Sciences, Institute of Public Health, University of Gondar, Gondar, Ethiopia. ${ }^{3}$ Maternal and Child Health Directorate, Ministry of Health, Addis Ababa, Ethiopia. ${ }^{4}$ Averting Maternal Death and Disability, Columbia University, NY and FHI 360, Columbia, North Carolina, USA.

Received: 20 September 2018 Accepted: 20 November 2019

Published online: 29 November 2019

\section{References}

1. Levels \& Trends in Child Mortality Estimates: The UN Inter-agency Group for Child Mortality Estimation United Nations; 2017.

2. Campbell OM, Calvert C, Testa A, Strehlow M, Benova L, Keyes E, Donnay F, Macleod D, Gabrysch S, Rong L, Ronsmans C, Sadruddin S, Koblinsky M, Bailey P. The scale, scope, coverage, and capability of childbirth care. Lancet. 2016;388:2193-208.

3. Central Statistical Agency [Ethiopia] and ICF International. Ethiopia. Demographic and Health Survey 2016. Addis Ababa, Ethiopia and Calverton, Maryland, USA.

4. The Federal Democratic Republic of Ethiopia Health Sector Transformation Plan: 2015/16-2019/20. Federal Ministry of Health, Addis Ababa, Ethiopia.

5. Mirkuzie AH, Sisay MM, Reta AT, Bedane MM. Current evidence on basic emergency obstetric and newborn care services in Addis Ababa, Ethiopia; a cross sectional study. BMC Pregnancy Childbirth. 2014;14:354.

6. Lee AC, Cousens S, Darmstadt GL, Blencowe H, Pattinson R, Moran NF, Hofmeyr GJ, Haws RA, Bhutta SZ, Lawn JE. Care during labor and birth for the prevention of intrapartum-related neonatal deaths: a systematic review and Delphi estimation of mortality effect. BMC Public Health. 2011;11(Suppl 3):S10.

7. Traoré M, Arsenault C, Schoemaker-Marcotte C, Coulibaly A, Huchon C, Dumont A, Fournier P. Obstetric competence among primary healthcare workers in Mali. Int J Gynecol Obstet. 2014;126:50-5.

8. Huchon C, Arsenault C, Tourigny C, Coulibaly A, Traore M, Dumont A, Fournier $P$. Obstetric competence among referral healthcare providers in Mali. Int J Gynecol Obstet. 2014;126:56-9.

9. Cheptum JJ, Gitonga MM, Mutua EM, Mukui SJ, Ndambuki JM, Koima WJ, Hale I. Emergency obstetrics training improving skills among health Care Workers in Migori and Nyeri Counties, Kenya. Int J Pharm Sci Res. 2016;7(7): 3045-52.

10. The Eclampsia Trial Collaborative Group. Which Anticonvulsant for Women with Eclampsia? Evidence from the Collaborative Eclampsia Trial. Stud Fam Plan. 1995;26(6):373.

11. Hafeez A. Barriers to the use of magnesium sulphate in Pakistan: a study to develop informed policy. Access to Medicines Policy Research; Ministry of Health, Pakistan. 2010. http://www.who.int/alliance-hpsr/projects/ alliancehpsr_magnesiumsulfate_pakistan.pdf. Accessed 2 May 2018.

12. Human Resources for Sexual and Reproductive Health Care. Maternal mortality: Nigeria, Burkina Faso and Pakistan. Reprod Health Matters. 2006; 14(27):219.

13. Homaifar N, Mwesigye D, Tchwenko S, Worjoloh A, Joharifard S, Kyamanywa P, Wilkinson J, Rulisa S, Thielman NM. Emergency obstetrics knowledge and practical skills retention among medical students in Rwanda following a short training course. Int J Gynaecol Obstet. 2013;120(2):195-9.

14. Ifeanyichukwu OA, Obehi OH. Knowledge of birth preparedness and complication readiness and associated factors among health care providers in Benin City, Edo State. J Pregnancy Child Health. 2016;3:278.

\section{Publisher's Note}

Springer Nature remains neutral with regard to jurisdictional claims in published maps and institutional affiliations.

\section{Ready to submit your research? Choose BMC and benefit from:}

- fast, convenient online submission

- thorough peer review by experienced researchers in your field

- rapid publication on acceptance

- support for research data, including large and complex data types

- gold Open Access which fosters wider collaboration and increased citations

- maximum visibility for your research: over $100 \mathrm{M}$ website views per year

At $\mathrm{BMC}$, research is always in progress.

Learn more biomedcentral.com/submissions 